\title{
Elimination of network intrusions via a resource oriented BDI architecture
}

https://doi.org/10.1515/comp-2018-0016

Received February 28, 2018; accepted October 8, 2018

\begin{abstract}
We propose a resource-oriented architecture of a rational agent for a network intrusion detection system. This architecture describes the behavior of a rational agent after detection of unwanted network activities. We describe the creation of countermeasures to ward off detected threats. Examples are created based on the proposed architecture, describing the process during a rational agent detection. We have described these examples by linear BDI logic behavioral formulæ, that have been proven by Gentzen sequent calculus.
\end{abstract}

Keywords: BDI logic, IDS, linear logic, resource oriented architecture

\section{Introduction}

Computer security and especially network security is a very recent research topic. The number of computers around us increases each day as they become a part of everyday life; consequently, there is a necessity to increase computer and network security to guard against malicious network activities.

Our recent research is dedicated to Intrusion Detection Systems (IDS), which can help make computer networks more secure. For that, we have created a real network laboratory environment, which consists of an attacker and a victim connected to a particular Local Area Network (LAN), and to the Internet. Based on our observations, we have design a verifiable formal coalgebraic model

^Corresponding Author: Ján Perháč: Department of Computers and Informatics, Faculty of Electrical Engineering and Informatics, Technical University of Košice, Košice, 04200 Košice, Slovak Republic; E-mail: Jan.Perhac@tuke.sk

Daniel Mihályi: Department of Computers and Informatics, Faculty of Electrical Engineering and Informatics, Technical University of Košice, Košice, 04200 Košice, Slovak Republic; E-mail: Daniel.Mihalyi@tuke.sk

Lukáš Mat’ǎ̌: Proxis, spol. s r.o., Bardejov, 08501 Bardejov, Slovak Republic; E-mail: Lukas.Matas@proxis.sk for the description of IDS's behavior [1]. With such a model, it will be possible to eliminate an undesirable behavior of IDS, and increase its efficiency. In [2], we have converted network signatures of real network attacks used by IDS to detect network intrusions [3], to coalgebraic signatures. In [4], we have used coalgebraic signatures to model IDS as a coalgebra of polynomial endofunctor over the category of infinite stream of packets. Therefore, we have first constructed the aforementioned category, and then we have specified a polynomial endofunctor over this category which formed a dynamics of the packet stream. In [5], we have specified the behavior of IDS during attacks as a formula of our modal linear logic. We have proven this formula by applying Gentzen sequent calculus rules. In [6], we have constructed a time-spatial structure of the mentioned formula from [5] using Girard's time-spatial theory called Ludics.

One of the future goals of our work is to extend IDS passive reactions at detected intrusions (expects system administrator intervention) to active ones [7]. To do so, we have chosen the Belief-Desire-Intention (BDI) logical system, together with linear logic [8-10]. In this paper, we define our linear BDI logic, and we propose a new BDI architecture for IDS. We show a motivating example of its behavior during two specific network intrusions, where we describe in detail how our architecture will behave. Firstly, we simulate a basic prerequisite attack technique for a "man-in-the-middle" type of attacks. Then, we describe its behavior by one popular "denial-of-service" type of attack. Both particular behaviors are expressed by corresponding provable logical formulæ.

\section{Basic notions from BDI}

A BDI logic controls decisions of BDI architecture agents. A BDI architecture is a philosophical theory of practical reasoning, originating on human reasoning and deduction based on the following attitudes: belief, desire, and intention [11]. Those attitudes represent mental situations that the BDI agent may acquire. The BDI agent is the "base unit" of the BDI architecture. It is based on the action chosen by 
the process of logical reasoning over gained beliefs about malicious network activities. This process consists of the following two steps:

1. we define the database of desires, based on the current belief of the agent,

2. we determine how specific actions will be met by actions from the intention database.

In terms of IDS, it is possible to use the BDI logic as follows: after gaining beliefs about (network) intrusion, it is possible to realize desires through intentions i.e. plans on how to create appropriate countermeasures. Principle of our goal is depicted in Figure 1.

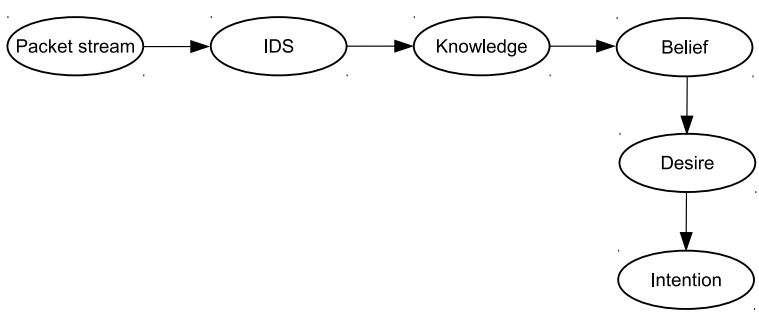

Figure 1: Usage of BDI logic to secure a computer system.

A BDI logic could be used not just as a formal specification language for agents [12], but also as a basic method of implementation of agents in BDI architectures [13]. Specifically, it is used for specification, design and verification of certain behavioral properties of agents.

A BDI logic introduces new modal connectives, that allow a description of a mental situation of an agent [14]. Generally, an agent can acquire the following mental situations:

- Belief. This situation expresses what information an agent possesses in the current state and in the world in which it exists.

- Desire. This situation expresses what the agent wants to achieve, i.e., which states will occur.

- Intention. This situation expresses what the agent can perform, i.e., the actions that will lead to the desires.

\subsection{Agents}

Nowadays, there is no simple or general definition of what an agent is. In many publications there is a plethora of different definitions and divisions of agents. For example, Wooldridge in [15] classifies agents to weak and strong based on specific properties that that agents possess. Another categorization of agent systems [16] is to single-agent systems and to multi-agent systems.
Based on autonomy [17], an agent can be described as an entity that works without direct human or other intervention and possesses control over its internal state. They can be divided as follows:

- Intelligent agent has ability to meet goals in its interest, using his own "intelligence," in most cases through adequate logical deduction.

- Reactive agent immediately responds to certain environmental changes without having an internal representation of the environment.

- Deliberative agent has ability to plan the progress of its actions leading to the achievement of the chosen goals or desires.

- Cognitive agent has the ability to deduce logical conclusions from its observations of the environment. In particular, such an agent must be able to learn and create its own knowledge.

- Rational agent possesses all of the above properties and its structure contains both a planning and a cognitive base including the knowledge base. It is an agent that, based on its own knowledge, is able to learn and then plan its activities to achieve its goals (desires).

Agent system is the set of the agents within the environment in which they operate. In our work, we use a singleagent system, with a rational agent [17]. It is able to learn and accordingly plan its next activities, to achieve its objectives.

\subsection{Architectures}

For BDI systems, there are several architectures such as: Jadex [18], PRS [19], or IRMA. The IRMA architecture is the first architecture mentioned with the approach based on BDI [20]. It is designed for planning and practical reasoning in the real environment, with limited time for decisionmaking and environmental knowledge about it. In this architecture, much attention is devoted to the problem of planning. Planning a solution consists of methods of environment examination and action sequencing, methods of examining the environment and action sequences which will yield numerous solutions to the final plan. A rational agent must consider must consider the final plan, alternative paths to achieving the plan, and appropriate communication during the process. Everything must be done while working with limited resources, and by performing calculations in a specific time. 


\subsection{BDI logic}

The original language of the BDI logic is based on the a $\mathcal{L}_{B D I}$ mentioned in [21]. It consists of the following:

- logical connectives of propositional logic:

$$
\neg(.), \wedge, \Rightarrow \text {, }
$$

- and modal connectives, which represent mental situations of an agent:

$$
B E L_{i}, D E S_{i}, I N T_{i}
$$

where $i$ represents the name of the agent.

Bratman et al. extended this language in their work [14] about temporal logical connectives of the Computation Tree Logic.

\section{Linear BDI logic}

Linear BDI logic (LBDI) is a logic of reasoning by a given agent based on resource oriented linear logic. In our approach, we define the language of the LBDI logical system as follows:

- We have translated logical connectives of the propositional logic into the multiplicative fragment of linear logic:

$$
(.)^{\perp}, 8, \otimes, \multimap
$$

- We have left the definitions of the BDI modal operators, $B E L_{i}, D E S_{i}$, and $I N T_{i}$ as is.

- Extension of temporal connectives is not necessary, because the linear logic's theory of Ludics came with the possibility that allows a description of logical time and space.

Our definition of LBDI language increases its expressive power. It can describe processes of the real-world such as causality, or resource consumption. Also, because of that, its syntax became quite simpler.

\subsection{Syntax of LBDI}

The syntax of the LBDI logical system can be expressed by following the production rule in Backus-Naur form:

$$
\begin{aligned}
\varphi::= & p|\mathbf{1}| \perp\left|\varphi^{\perp}\right| \varphi \otimes \varphi|\varphi \ngtr \varphi| \varphi \multimap \varphi \\
& \left|B E L_{i}(\varphi)\right| D E S_{i}(\varphi) \mid \operatorname{INT}_{i}(\varphi)
\end{aligned}
$$

For any $p, \varphi, \psi$ we formulate that:
- $\quad p$ is the elementary formula.

- $\quad 1$ and $\perp$ are neutral elements for $\otimes$ and 8 , respectively.

- $\varphi^{\perp}$ is the linear negation, which expresses duality between action $(\varphi)$ and reaction $\left(\varphi^{\perp}\right)$.

- $\varphi \otimes \psi$ is the multiplicative conjunction, which expresses the performing of both actions simultaneously.

- $\varphi \& \psi$ is the multiplicative disjunction, which expresses commutativity of duality between available and consumed resources by performing either action $\varphi$ or action $\psi$.

- $\quad \varphi \multimap \psi$ is the linear implication, which expresses that a (re)action $\psi$ is a causal consequence of action $\varphi$ and after performing this implication, the resource $\varphi$ became consumed $\left(\varphi^{\perp}\right)$.

- $B E L_{i}(\varphi)$ denotes that the agent $i$ believes that $\varphi$.

- $\operatorname{DES}_{i}(\varphi)$ denotes that the action $\varphi$ is the desire of the agent $i$.

- $\operatorname{INT}_{i}(\varphi)$ denotes that the action $\varphi$ is the intention of the agent $i$.

\subsection{Proof system of LBDI}

We define the proof system of the LBDI by Gentzen's sequent calculus. One can the define proof system through either the right side form or double side form:

$$
\vdash \varphi, \psi \Delta \quad \Gamma, \varphi \vdash \psi, \Delta
$$

$$
\text { right side sequent double sided sequent }
$$

where $\Gamma, \Delta$ are linear contexts for formulæ $\varphi, \psi$. We have chosen the double sided sequent form. The inference rules (without the weakening and contraction rules) for our LBDI are listed below.

1. The identity rule is an axiom i.e. is the only rule which has no assumptions. It expresses that action $\varphi$ you can prove reaction $\varphi$ :

$$
\overline{\varphi \vdash \varphi}(i d)
$$

2. Structural rules are a cut rule and the exchange rules:

$$
\frac{\Gamma \vdash \varphi \quad \Delta, \varphi \vdash \psi}{\Gamma, \Delta \vdash \psi}(\text { cut })
$$

Exchange rules express the logical property of commutativity by allowing the permutation of formulæ on both sides of the sequent:

$$
\frac{\Gamma, \varphi, \psi \vdash \Delta}{\Gamma, \psi, \varphi \vdash \Delta}\left(e x_{l}\right) \quad \frac{\Gamma \vdash \varphi, \psi, \Delta}{\Gamma \vdash \psi, \varphi, \Delta}\left(e x_{r}\right)
$$

3. Logical rules deal with logical connectives:

$$
\frac{\Gamma \vdash \Delta}{\Gamma, \mathbf{1} \vdash \Delta}\left(\mathbf{1}_{l}\right) \quad \overline{\vdash \mathbf{1}}^{\left(\mathbf{1}_{r}\right)} \quad \overline{\perp \vdash}^{\left(\perp_{l}\right)} \frac{\Gamma \vdash \Delta}{\Gamma \vdash \perp, \Delta}\left(\perp_{r}\right)
$$




$$
\begin{gathered}
\frac{\Gamma, \varphi, \psi \vdash \Delta}{\Gamma, \varphi \otimes \psi \vdash \Delta}\left(\otimes_{l}\right) \quad \frac{\Gamma_{1} \vdash \varphi, \Delta_{1} \quad \Gamma_{2} \vdash \psi, \Delta_{2}}{\Gamma_{1}, \Gamma_{2} \vdash \varphi \otimes \psi, \Delta_{1}, \Delta_{2}}\left(\otimes_{r}\right) \\
\frac{\Gamma \vdash \varphi, \Delta \quad \Phi, \psi \vdash \Sigma}{\Gamma, \Phi, \varphi \multimap \psi \vdash \Delta, \Sigma}\left(-_{l}\right) \quad \frac{\Gamma, \varphi \vdash \psi, \Delta}{\Gamma \vdash \varphi \multimap \psi, \Delta}\left(-_{r}\right) \\
\frac{\Gamma, \varphi \vdash \Delta \quad \Phi, \psi \vdash \Sigma}{\Gamma, \Phi, \varphi \ngtr \psi \vdash \Delta, \Sigma}\left(8_{l}\right) \quad \frac{\Gamma \vdash \varphi, \psi, \Delta}{\Gamma \vdash \varphi \ngtr \psi, \Delta}\left(8_{r}\right) \\
\frac{\Gamma \vdash \varphi, \Delta}{\Gamma, \varphi^{\perp} \vdash \Delta}\left(0_{l}^{\perp}\right) \quad \frac{\Gamma, \varphi \vdash \Delta}{\Gamma \vdash \Delta, \varphi^{\perp}}\left(0_{r}^{\perp}\right)
\end{gathered}
$$

4. In [22] Nide and Takata introduced the inference rules to deal with modal BDI logical connectives:

$$
\begin{gathered}
\frac{\Gamma \vdash \varphi}{B E L_{i}(\Gamma) \vdash B E L_{i}(\varphi)}\left(B E L_{i}\right) \\
\frac{\Gamma \vdash \varphi}{D E S_{i}(\Gamma) \vdash D E S_{i}(\varphi)}\left(D E S_{i}\right) \\
\frac{\Gamma \vdash \varphi}{I N T_{i}(\Gamma) \vdash I N T_{i}(\varphi)}\left(I N T_{i}\right)
\end{gathered}
$$

\section{Design of resource oriented BDI architecture for IDS}

Our resource-oriented BDI architecture for IDS is based on the IRMA. It is designed for planning and practical decision-making of a rational agent after the detection of an intrusion at the computer network. High attention is paid to the problem of planning, where the sequence of actions, which will be performed after detected the network intrusion, is chosen.

\subsection{Structure of resource oriented BDI architecture for IDS}

The main structures of our architecture are following:

- Plan library are sequences of actions performed after detecting the network intrusion.

- Beliefs represent the current situation of the agent that is convinced of an intrusion at the computer network. The knowledge about the intrusion, which implies the belief, is acquired by analyzing the network traffic and the following warning about performed intrusion by IDS.

- Desires represent the agent's motivation to hold defined security rules. These are the goals of a rational agent to implement a plan by which an intrusion will be eliminated based on an organisation's security policy.

- Intentions represent the situation of the agent, when it is already determined that elimination of a network intrusion is necessary based on violated security rules. It is a situation when the agent is convinced that the proposed plan is appropriate to eliminate the intrusion.

- IP database is a list of IP addresses from which intrusions were made.

- Administrator is an e-mail account for alerting the administrator about the treated intrusion.

- FW database is a rule database, from where a particular rule is added to the firewall.

- End of plan serves for terminating and disabling access to the host network.

The main aspects of our architecture to achieve rational agent plans are the following:

- Planning process is a process when a rational agent creates a plan by comparing rational belief about a realization of an intrusion using the IP address database and the firewall rules.

- Opportunity analyzer is a process where a rational agent decides about the believed options, and the options that it wants to include in the plan.

- Filtering process is a process in which an agent reevaluates current plans with propositions to change the plans that were proposed in the Opportunity analyzer.

The design of our Resource-Oriented BDI Architecture for IDS (ROAIDS) is depicted in Figure 2.

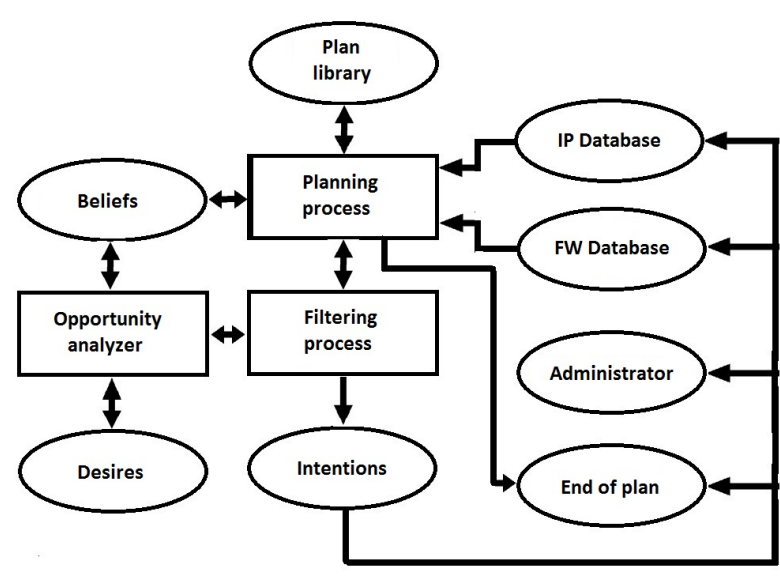

Figure 2: Resource-oriented BDI architecture for IDS. 


\subsection{Behavior of ROAIDS during port scan intrusion}

In this motivating example, we describe a behavior of our architecture during Port Scan intrusion. This intrusion is a very popular prerequisite attack technique. It is designed to detect running services as open ports [23]. All computers connected to a LAN or the Internet have numerous services running i.e. have some ports enabled. Port scanning consists of sending messages to all ports, or selected ports within a specific range. Then, depending on the type of the response, the attacker can determine which ports are opened and which are not. Based on this (important) information, the attacker can focus their attacks at the specified ports and services by exploiting known errors.

A key aspect of our architecture is the Plan library, which holds the sequence of actions that are to be performed for identifying and eliminating detected network intrusion.

The Plan library contains a following sequence of steps for a rational agent:

1. Analysis of network traffic - In this step the architecture watches for a pattern of known types of network intrusions. If a suspicious pattern is detected, it will $\log$ an alert about realization of intrusion. Let it be denoted as $\varphi$.

2. Addition of IP address into IP database - This step will add an IP address of the attacker in to the IP database. Let it be denoted as $\psi$.

3. Creation and addition of firewall rules - This will create appropriate firewall rules and add them into the firewall rule database. Let it be denoted as $\gamma$.

4. Alert of administrator - In case a port scan intrusion is detected. This step will send information abouttype of intrusion and attacker identity to the administrator. Let it be denoted as $\delta$.

5. End of plan - In the last step, the architecture terminates the execution of a rational agent after intrusion detection and the execution of all necessary countermeasures. Let it be denoted as $\vartheta$.

The first phase of our logical reasoning begins with detecting an intrusion $\varphi$ by the coalgebra of polynomial endofunctor as IDS through coalgebraic modal linear logic [5] over category of the infinite stream of packets [4], the semantics of which were defined by the Kripke model in time spatial proof-tree structure [6]. This phase is described by our verifiable model of IDS's behavior in [1].

The second phase involves obtaining the knowledge $K_{a} \varphi$ about detected intrusion $\varphi$ based on significant symptoms from the (network) signatures [2] using the Epis- temic linear logic [24]. This process ends by the fact that objective knowledge implies the rational belief of the rational agent, i.e. $K_{a} \varphi \multimap B E L_{a} \varphi$.

Now, we are interested in next phase with input $B E L_{a} \varphi$, where the planning process begins by choosing an appropriate plan from the BDI Architectures Plan library by comparing the obtained belief of the rational agent $B E L_{a} \varphi$, together with the IP database $\psi$ and the FW database $\gamma$.

The situation where the attackers' IP address does not match with the IP databases' addresses, and where the firewall does not contain appropriate rules for eliminating Port Scan intrusion $\gamma^{\perp}$, can be expressed by the following formula of LBDI logic:

$$
B E L_{a} \varphi \otimes \psi^{\perp} \otimes \gamma^{\perp}
$$

We use the GSK defined in the section 3.2. Proof of the formula in GSK is a proof tree, where the root of this tree is the sequent containing the formula. Every step of deduction is created by using an appropriate rule, until all leaves are identities (axioms).

The sequent in the root of the proof tree is defined as follows: the left side of the sequent contains the treated formula, and the right side contains the necessary resources constructed by elaborating the proof tree of the formula on the left side. Every application of the appropriate rule describes the behavior of the architecture (IDS) stepwise, during the specific situation e.g. dealing with intrusion. The proof tree is depicted in Figure 3.

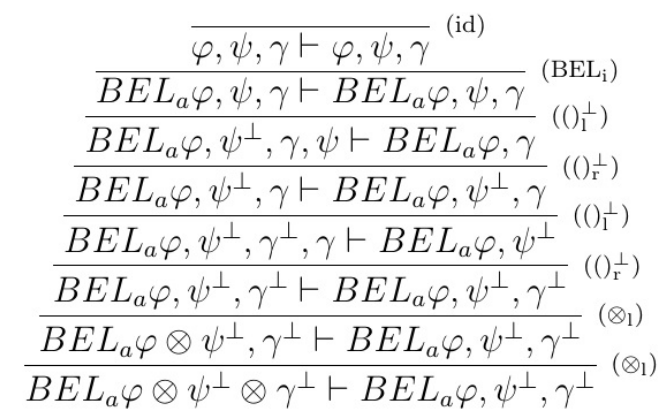

Figure 3: Proof tree.

Rational agent $a$ will send a plan to the Filtering process, where it will be compared by the Opportunity analyzer. Then, during the process of opportunity analysis, the rational agent will compare its belief about the Port Scan $B E L_{a} \varphi$ to comply with the rational agent's desires. In this case, the desires are as following:

- Add the IP address of attacker into the IP database: $D E S_{a} \psi$. 
- Create appropriate firewall rules and add them into FW database: $D E S_{a} \gamma$.

- Alert administrator about realization of Port Scan: $D E S_{a} \delta$.

- End plan: $D E S_{a} \vartheta$.

The aforementioned process can be described by the following formula of LBDI logic:

$$
B E L_{a} \varphi \otimes D E S_{a} \psi \otimes D E S_{a} \gamma \otimes D E S_{a} \delta \otimes D E S_{a} \vartheta
$$

After comparing the rational agent's beliefs and desires by the Opportunity analyzer, the agent will send a plan to the Filtering process. Based on the results from the planning process and opportunity analyzer, the plan will be reevaluated. This process can be expressed by the following formula of LBDI logic:

$$
\begin{gathered}
\left(B E L_{a} \varphi \otimes \psi^{\perp} \otimes \gamma^{\perp}\right) \otimes \\
\left(B E L_{a} \varphi \otimes D E S_{a} \psi \otimes D E S_{a} \gamma \otimes D E S_{a} \delta \otimes D E S_{a} \vartheta\right)
\end{gathered}
$$

After that, the rational agent will send the plan to the Intentions structure, where the following intentions will be created:

- Add the IP address of an attacker into the IP database: $I N T_{a} \psi$.

- Add firewall rules into the FW database: $I N T_{a} \gamma$.

- Alert administrator: $I N T_{a} \delta$.

- End plan: INTa 9 .

The process of creating desires to eliminate network intrusion can be expressed by following formula of the LBDI logic:

$$
\begin{gathered}
\left(\left(B E L_{a} \varphi \otimes \psi^{\perp} \otimes \gamma^{\perp}\right)\right. \\
\otimes \\
\left.\left.\left(B E L_{a} \varphi \otimes D E S_{a} \psi \otimes D E S_{a} \gamma \otimes D E S_{a} \delta \otimes D E S_{a} \vartheta\right)\right)\right) \\
\multimap \\
\left(I N T_{a} \psi \otimes I N T_{a} \gamma \otimes I N T_{a} \delta \otimes I N T_{a} \vartheta\right)
\end{gathered}
$$

A plan is realized according to the created desires as follows:

- Add the IP address of an attacker into the IP database: $\psi$.

- Add firewall rules into the FW database: $\gamma$.

- Alert administrator: $\delta$.

- End plan: $\vartheta$.

The whole process of realizing a plan for identification and elimination of the network intrusion can be expressed by the following formula of the LBDI logic:

$$
\begin{gathered}
\left(\left(\left(B E L_{a} \varphi \otimes \psi^{\perp} \otimes \gamma^{\perp}\right) \otimes\right.\right. \\
\left.\left(B E L_{a} \varphi \otimes D E S_{a} \psi \otimes D E S_{a} \gamma \otimes D E S_{a} \delta \otimes D E S_{a} \vartheta\right)\right) \multimap \\
\left.\left(I N T_{a} \psi \otimes I N T_{a} \gamma \otimes I N T_{a} \delta \otimes I N T_{a} \vartheta\right)\right) \multimap \\
(\psi \otimes \gamma \otimes \delta \otimes \vartheta)
\end{gathered}
$$

The behavior of our architecture during a situation when the attacker tries to perform a port scan from the same or a different IP address is as follows:

- The plan library has the same sequence of actions as in the case of first Port Scan.

- The planning process. Agent shall process its rational belief about the intrusion $B E L_{a} \varphi$.

- The agent will compare the attacker's IP address to IP database $\psi$.

- Where a match is not found, the agent will check whether the FW database contains appropriate firewall rules $\gamma$.

The behavior of the architecture after a repeated port scan network intrusion can be expressed by the following formula of the LBDI logic:

$$
\left(B E L_{a} \varphi \otimes(\psi 8 \gamma)\right) \multimap \vartheta
$$

with the following proof tree depicted in Figure 4.

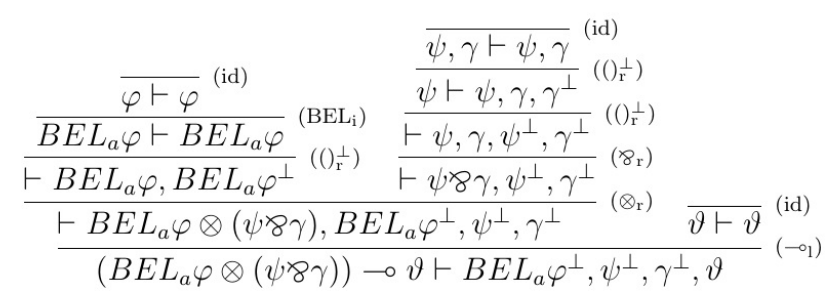

Figure 4: Proof tree.

\subsection{Behavior of ROAIDS during syn-flood attack}

The SYN-flood attack belongs to a Denial of Service family of attacks. This attack exploits the TCP three-way handshake. In brief, the principle of the TCP three-way handshake is following:

1. a client sends a SYN request,

2. a server responds with SYN-ACK to a client,

3. a client sends back ACK, and a connection is established. 
During a SYN-flood attack, an attacker sends a succession of SYN requests to targets's system, but with spoofed IP addresses, therefore a server won't receive an ACK from a client. A server will wait for a response for some time, during which an attacker flood a server with requests which will cause its malfunction.

After our architecture gains a belief about realization of a SYN-flood attack, the plan library possesses a similar sequence of actions for elimination of this attack as it was in the Port-Scan case. But now the architecture won't compare an IP address with IP addresses in the IP database, because the attacker has sends spoofed IP addresses. Therefore the planning process checks if there are appropriate firewall rules $\gamma$ in the $F W$ database. If the SYNflood attack was performed for the first time, it will determine that there are no FW rules $\gamma^{\perp}$. This process is described by the following formula:

$$
B E L_{a} \varphi \otimes \gamma^{\perp}
$$

with the proof tree depicted in Figure 5.

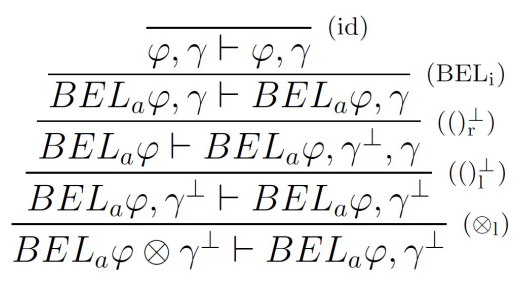

Figure 5: Proof tree.

Rational agent $a$ will send a plan to the Filtering process, where it will be compared by the Opportunity analyzer. Then, during the process of opportunity analysis, the rational agent will compare its belief about the SYN-flood $B E L_{a} \varphi$ to comply with the desires of the rational agent. In this case, the desires are following:

- Create appropriate firewall rules and add them into FW database: $D E S_{a} \gamma$.

- End plan: DESa .

This process is described by the following formula:

$$
B E L_{a} \varphi \otimes D E S_{a} \gamma \otimes D E S_{a} \vartheta
$$

with the proof tree depicted in Figure 6.

After comparing rational agents beliefs and desires by the Opportunity analyzer, the agent will send a plan to the Filtering process. Based on the results from the planning process and Opportunity analyzer, the plan will be reevaluated. This process can be expressed by the following for-

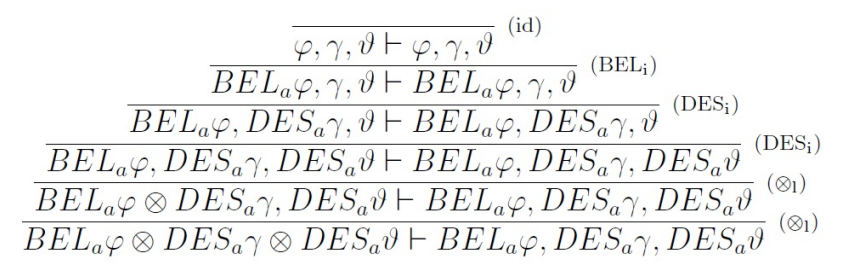

Figure 6: Proof tree.

mula of LBDI logic:

$$
\left(B E L_{a} \varphi \otimes \gamma^{\perp}\right) \otimes\left(B E L_{a} \varphi \otimes D E S_{a} \gamma \otimes D E S_{a} \vartheta\right)
$$

After that, the rational agent will send the plan to the Intentions structure, where the following intentions will be created:

- Add firewall rules into the FW database: $I N T_{a} \gamma$.

- End plan: $I N T_{a} \vartheta$.

The process of creating desires to eliminate network attack can be expressed by following formula of the LBDI logic:

$$
\begin{gathered}
\left(\left(B E L_{a} \varphi \otimes \gamma^{\perp}\right) \otimes\left(B E L_{a} \varphi \otimes D E S_{a} \gamma \otimes D E S_{a} \vartheta\right)\right) \\
\multimap \\
\left(I N T_{a} \gamma \otimes I N T_{a} \vartheta\right)
\end{gathered}
$$

A plan is realized according to the created desires as follows:

- Add firewall rules into the FW database: $\gamma$.

- End plan: $\vartheta$.

The whole process of realizing a plan for identifying and eliminating the network attack can be expressed by the following formula of the LBDI logic:

$$
\begin{gathered}
\left(\left(B E L_{a} \varphi \otimes \gamma^{\perp}\right) \otimes\left(B E L_{a} \varphi \otimes D E S_{a} \gamma \otimes D E S_{a} \vartheta\right)\right) \\
\multimap \\
\left(I N T_{a} \gamma \otimes I N T_{a} \vartheta\right) \multimap(\gamma \otimes \vartheta)
\end{gathered}
$$

During the second attempt to perform the SYN-flood attack our architecture will behave as follows. A Plan library possesses the same sequence of actions. In the Planning process a rational agent will compare its rational belief about attack $B E L_{a} \varphi$, with rules in the $F W$ database $\gamma$. In this case, the firewall contains appropriate rules to eliminate the attack, therefore it will perform the end of plan action $\vartheta$. This process can described by the following LBDI formula:

$$
\left(B E L_{a} \varphi \otimes \gamma\right) \multimap \vartheta
$$

with the proof tree depicted in Figure 7. 


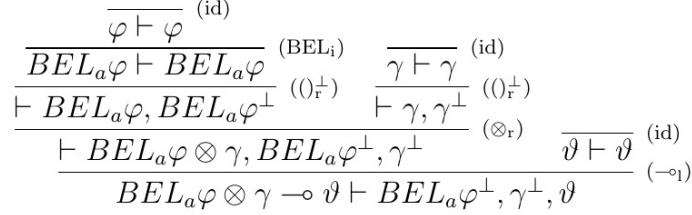

Figure 7: Proof tree.

\section{Conclusion}

In this paper, we propose a resource oriented BDI architecture for IDS. Because of that, we have created its controlling logical system based on the BDI logic and linear logic. We have simulated a Port Scan intrusion, a SYN-flood attack, and then we have described the behavior of this architecture stepwise. Every step is described by a provable formula of our LBDI logic. Here, we present only few proofs in Gentzen sequent calculus, because a few proofs of larger formulæ are rather complex.

The design of our architecture allows automated response of the IDS to incoming intrusions. In the future, we would like to extend our approach with implementation of our architecture into a real network environment. We also plan to combine a network intrusion detection system with a host-based intrusion detection system. This would further increase the complex security of our computer system.

Acknowledgement: This work was supported by the following projects:

- Faculty of Electrical Engineering and Informatics, Technical University of Košice under the contract No. FEI-2017-47: Design and development of verifiable BDI architecture for IDS using component and virtual reality systems;

- International cooperation under the CEEPUS network No. CIII-HU-0019-12-1617;

- Slovak Research and Development Agency under the contract No. SK-AT-2017-0012: "Semantics technologies for computer science education".

\section{References}

[1] Perháč J., Mihályi D., Novitzká V., Design of verifiable model of program systems' complex security using coalgebras and coalgebraic logics, In: Electrical Engineering and Informatics 7: proceedings of the Faculty of Electrical Engineering and Informatics of the Technical University of Košice, Košice, FEEI TU, 2016, 120-124
[2] Perháč J., Mihályi D., Coalgebraic specification of network intrusion signatures, Studia Universitatis Babes-Bolyai, Informatica, 2016, 61(3), 83-94

[3] Roesch M., Snort users manual, 2016, https://www.snort.org/

[4] Perháč J., Mihályi D., Coalgebraic modeling of IDS behavior, 13th International Scientific Conference on Informatics, November 18-20, 2015, Poprad, Slovakia, Danvers: IEEE, 2015, 201-205

[5] Perháč J., Mihályi D., Intrusion detection system behavior as resource-oriented formula, Acta Electrotechnica et Informatica, 2015, 15(3), 9-13

[6] Perháč J., Mihályi D., Novitzká V., Between syntax and semantics of resource oriented logic for IDS behavior description, Journal of Applied Mathematics and Computational Mechanics, 2016, 15(2), 105-118

[7] Perháč J., Mihályi D., Mataš L., Resource oriented BDI architecture for IDS, In: Proceedings of the 14th International Conference on Informatics, Informatics 2017, November 14-16, 2017, Poprad, Slovakia, IEEE, 2017, 293-298

[8] Novitzká V., Mihályi D., Slodičák V., Linear logical reasoning on programming, Acta Electrotechnica et Informatica, 2006, 6(3), 34-39

[9] Steingartner W., Polákova A., Pražnák P., Novitzká V., Linear logic in computer science, Journal of Applied Mathematics and Computational Mechanics, 2015, 14(1), 91-100

[10] Steingartner W., Radaković D., Novitzká V., Eldojali M., An analysis of some aspects of component-based programming for selecting appropriate categorical structures as their models, Acta Electrotechnica et Informatica, 2017, 17(2), 3-10

[11] Wooldridge M. J., Reasoning about rational agents, MIT press, 2000

[12] Emerson E. A., Srinivasan J., Branching time temporal logic, In: Workshop/School/Symposium of the REX Project, Research and Education in Concurrent Systems, Springer, 1988, 123-172

[13] Singh M. P., Rao A. S., Georgeff M. P., Formal methods in DAl, Logic-based representation and reasoning, Multiagent Systems, MIT Press, 1991, 331-376

[14] Bratman M. E., Israel D. J., Pollack M. E., Plans and resourcebounded practical reasoning, Computational Intelligence, 1988, 4(3), 349-355

[15] Wooldridge M., Jennings N. R., Intelligent agents: Theory and practice, The knowledge engineering review, 1995, 10(2), $115-152$

[16] Wooldridge M., Jennings N. R., Agent theories, architectures, and languages: a survey, International Workshop on Agent Theories, Architectures, and Languages, Springer, 1994, 1-39

[17] Kubík A., Inteligentní agenty: tvorba aplikačního software na bázi multiagentových systému, Computer Press, 2004

[18] Pokahr A., Braubach L., Lamersdorf W., Jadex: A BDI reasoning engine, Multi-agent Programming, 2005, 149-174

[19] Georgeff M. P., Lansky A. L., Reactive reasoning and planning, In: AAAI, 1987, 87, 677-682

[20] Kubík A., Agenty a multiagentové systémy, Slezská univerzita, Filozoficko-přírodovědecká fakulta, Ústav informatiky, 2000

[21] Nair V., On Extending BDI Logics, Griffith University, 2003

[22] Naoyuki N., Takata S., Deduction systems for BDI logics using sequent calculus, In: Proceedings of the first international joint conference on Autonomous Agents and Multiagent Systems: part 2, ACM, 2002, 928-935 
[23] de Vivo M., Carrasco E., Isern G., de Vivo G. O., A review of port scanning techniques, SIGCOMM Comput. Commun. Rev., 1999, 29(2), 41-48
[24] Mihályi D., Novitzká V., Towards the knowledge in coalgebraic model of IDS, Computing and Informatics, 2014, 33(1), 61-78 